\title{
Extension of Bayesian inference for multi-experimental and coupled problem in neutronics - a revisit of the theoretical approach
}

\author{
Thomas Frosio*, Thomas Bonaccorsi, and Patrick Blaise \\ French Atomic Energy and Alternative Energies Commission CEA, DEN, CAD, Reactor Studies Department, \\ 13108 Saint Paul-Lez-Durance, France
}

Received: 4 February 2018 / Received in final form: 22 April 2018 / Accepted: 9 July 2018

\begin{abstract}
Bayesian methods are known for treating the so-called data re-assimilation. The Bayesian inference applied to core physics allows to get a new adjustment of nuclear data using the results of integral experiments. This theory leading to reassimliation encompasses a broader approach. In previous papers, new methods have been developed to calculate the impact of nuclear and manufacturing data uncertainties on neutronics parameters. Usually, adjustment is performed step by step with one parameter and one experiment by batch. In this document, we rewrite Orlov theory to extend to multiple experimental values and parameters adjustment. We found that the multidimensional system expression looks like can be written as the monodimensional system in a matrix form. In this extension, correlation terms appears between experimental processes (manufacturing and measurements) and we discuss how to fix them. Then formula are applied to the extension to the Boltzmann/Bateman coupled problem, where each term could be evaluated by computing depletion uncertainties, studied in previous papers.
\end{abstract}

\section{Introduction}

The required accuracies on the nuclear data are difficult to reach using only differential experiments, even if innovative experimental techniques are used. The use of integral experiments has been essential in the past to ensure enhanced predictions for power fast reactor cores. In some cases, these integral experiments have been documented in an effective manner and the associated uncertainties are well understood. A combined use of scientifically based covariance data and of integral experiments can be made using advanced statistical adjustment techniques (see, e.g. [1-4]). These techniques can provide in a first step adjusted nuclear data $[5,6]$ for a wide range of applications with new and improved covariance data and bias factors (with reduced uncertainties) for the required design parameters, in order to meet design target accuracies. Moreover, the purpose of cross sections adjustment is more and more perceived as of providing useful feedback to evaluators and differential measurement experimentalists. It then allows to improve the knowledge of neutron cross sections to be used in a wider range of applications.

\footnotetext{
* e-mail: thomas.frosio@gmail.com
}

However, results concerning the nuclear data propagation on GEN-II and GEN-III reactor parameters, and even more for MTR are scarce: if uncertainty quantification methods are well established for Boltzmann problem, as well as separated Bateman problems, an accurate and rigorous treatment of nuclear data uncertainty propagation in coupled problem is still missing.

An important gap between "step 0" uncertainty calculation and depletion uncertainty calculation must then be filled. The major unknown, uncertainties on isotopes concentrations in the reactor core can be estimated by decorrelating sources of uncertainties.

The large amount of integral experiments performed on different nuclear reactors could be taken into account to improve the knowledge of the nuclear data and covariance matrix, in addition to nuclear data differential experiments. These integral experiments could be based on different integral parameters such as reactivity but also radionuclide concentrations, power distribution or reactivity coefficients. They could be simultaneously used for nuclear data adjustment as we will explain in this document.

After the description of the representativity theory, we extend the concept to multiple experiments and then we take into account Boltzmann/Bateman coupled problem. 


\section{State of art}

Sensitivity coefficients and uncertainties can be used to adjust nuclear data and have a feedback on neutronics parameters of interest from integral experiments. An example of adjustment with all the necessary steps (sensitivity analysis, interest parameters uncertainties, experiment analysis) can be found in [7].

We consider a nuclear data set $\sigma_{0}$ associated to a covariance matrix $M_{\sigma}$ from a data evaluation. The representativity or data adjustment has been introduced by Usachev [8]. This method allows to reduce the uncertainties specified in $M_{\sigma}$ called "a priori" to get a new "a posteriori" covariance matrix $M_{\sigma}^{*}$ taking into account the information contained in the experiments as used in [9] for super-Phenix reactor. This covariance matrix is associated to $\sigma^{*}$ the posterior nuclear data re-evaluated during the same process of adjustment.

Considering $C$ parameters of interest calculated with a simulation code, $S_{C}$ their sensitivity matrix to the $\sigma_{0}$ nuclear data, the covariance matrix $M_{c}$ of parameters of interest can be written as:

$$
M_{C}=S_{C} M_{\sigma} S_{C}^{T}
$$

One of the important hypothesis done for this equation (1) is based on a first order Taylor expansion to linearize the $C$ parameters for small nuclear data perturbations around their expectancies. This can be translated by equation (2):

$$
C\left(\sigma^{*}\right) \approx C+S_{C} \cdot\left(\sigma^{*}-\sigma_{0}\right) .
$$

$C\left(\sigma^{*}\right)$ refers to the value of $C$ computed with $\sigma^{*}$ and $C$ is linked to $\sigma_{0}$.

Different reasoning ways [10] can give the next equation (3). Considering this set of integral experiments measured in $E$ experiments, we can show using Bayes theorem and maximizing the likelihood, with generalized least-squares, and applying Sherman-Morrison-Woodbury formulation of matricial inverse:

$$
\left\{\begin{array}{l}
\sigma^{*}-\sigma_{0}=M_{\sigma} S_{E}^{T}\left[M_{E}+S_{E} M_{\sigma} S_{E}^{T}\right]^{-1}(E-C) \\
M_{\sigma}^{*}=M_{\sigma}-M_{\sigma} S_{E}^{T}\left[M_{E}+S_{E} M_{\sigma} S_{E}^{T}\right]^{-1} S_{E} M_{\sigma}
\end{array}\right.
$$

with:

- $M_{\sigma}$ is the covariance matrix (COMAC for example) $[11,12]$ on nuclear data. The dimension of $M_{\sigma}$ is $q \times q$.

- $\sigma_{0}$ is the evaluation vector of the nuclear data (prior). The dimension of $\sigma_{0}$ is $q$.

- $C$ is the integral parameters vector calculated with a simulation code and with the $\sigma_{0}$ nuclear data. The dimension of $C$ is $p$.

- $E$ is the experimental vector of the integral parameters. The dimension of $E$ is $p$.

- $S_{C}$ is the sensitivity matrix of the $C$ interest parameters to $\sigma_{0}$. The dimensions of $S_{C}$ is $q \times p$.

- $S_{E}$ is the sensitivity matrix of the $E$ interest parameters to $\sigma_{0}$. The dimensions of $S_{E}$ is $q \times q$.

- $M_{E}$ is the covariance matrix associated to the integral parameters observed in the $E$ experiments.
The dimension of $M_{E}$ is $p \times p$, and represents the experimental uncertainty. In a general meaning, $M_{E}$ defined as the experimental uncertainty represents:

- the knowledge we have about the results given by the measurement (uncertainty of the measurement device or measurement process);

- the knowledge we have on the manufacturing parameters such as geometry, position of assemblies, enrichment, mass balance.

$-\sigma^{*}$ is the adjuster vector of the nuclear data (posterior). The dimension of $\sigma^{*}$ is $q$.

$-M^{*}$ is the new covariance matrix associated to $\sigma^{*}$. The dimension of $M^{*}$ is $q \times q$.

$\sigma^{*}$ and $M^{*}$ are built during the same mathematical process and associated in a system of equations. They are fully linked and cannot be used independently.

$S_{C}$ and manufacturing parameters from $M_{E}$ can be obtained following the methods described in [13-15].

The transposition is an application of the representativity. It allows to transmit the result of an experiment $E$ modelled in a calculation code $C$ to another application $R$ evaluated with a calculation code in case that the representativity between the experience and the other application is high (close to 1 ).

The combination of equations (1) and (2) with (3) gives:

$$
\left\{\begin{array}{l}
R^{*}-R=S_{R} M_{\sigma} S_{E}^{T}\left[M_{E}+S_{E} M_{\sigma} S_{E}^{T}\right]^{-1}(E-C) \\
M_{R}^{*}=S_{R} M_{\sigma} S_{R}^{T}-S_{R} M_{\sigma} S_{E}^{T}\left[M_{E}+S_{E} M_{\sigma} S_{E}^{T}\right]^{-1} S_{E} M_{\sigma} S_{R}^{T}
\end{array}\right.
$$

with $R^{*}$ represents the integral parameters estimated for another design with the adjusted set $\sigma^{*}$ of nuclear data and $M_{R}^{*}$ is the posterior covariance matrix for the integral parameters of $R$. We recognize $M_{R}=S_{R} M_{\sigma} S_{R}^{T}$ the a priori covariance matrix of the integral parameters of $R$.

Considering one integral experiment and one parameter of interest associated to this experiment, we find the Orlov formulation [1]:

$$
\left\{\begin{array}{l}
R^{*}-R=\omega r_{E, R}(E-C) \frac{\varepsilon(R)}{\varepsilon(E)} \\
\varepsilon^{* 2}(R)=\varepsilon^{2}(R)-\varepsilon^{2}(R) \omega r_{E, R}^{2}
\end{array}\right.
$$

with:

- $r_{E, R}$ the representativity between the reactor and the experiment $E$ for the neutronics parameter $R$. It is a Pearson correlation coefficient;

- $\omega$ is a neutronics weight describing the quality of the experiment. $\varepsilon^{2}(R)$ and $\varepsilon^{* 2}(R)$ are respectively the uncertainties a priori and a posteriori of the parameter $R$. It provides information on the contribution of the experiment in terms of uncertainty knowledge.

These values are given as:

$$
\left\{\begin{array}{l}
r_{E, R}=\frac{S_{R} M_{\sigma} S_{E}^{T}}{\varepsilon(R) \varepsilon(E)} \\
\omega=\frac{\varepsilon^{2}(E)}{\varepsilon^{2}(E)+\delta^{2}}
\end{array}\right.
$$


with:

$-\varepsilon(X)$ representing the standard deviation of $X, X$ being any parameter;

$-\delta^{2}$ represents the experimental uncertainty. We use this notation instead of $\varepsilon$ to not overload the equations with indices to distinguish nuclear data uncertainties and experimental uncertainties.

\section{Extension to multiple experiments}

In the following, we will work with the transposition process starting with equations (4). However, the nuclear data adjustment is following exactly the same assumptions and transformations, starting with equation (3) instead of (4). A work has already been done in [17] to consider different experiments for a set of nuclear data, but we want here to give details on the experimental covariance matrix $M_{E}$ and express experimental correlations.

In the case where experiments are performed for example on local neutronics parameters such as power factors, or reactivities from different benchmark, the number of experimental results can be important. This is the reason why we now want to extend the data assimilation to cases with multiple experiments and for different neutronics parameters. These parameters can be those studied in [13] and [14]: isotopic concentrations, power factors and reactivity. Different design studies and different experiments not necessarily correlated can be used for the adjustment. Starting from equation (4), we will express $\left[M_{E}+S_{E} M_{\sigma} S_{E}^{T}\right]^{-1}$. This matrix is depending on the experimental data base. We need to express $M_{E}$ and will use the methodology proposed by Dos Santos [18] for this purpose.

\subsection{Expression of experimental correlations}

$M_{E}$ is a matrix containing the experimental sources of uncertainties that we will write $\delta^{2}$ to not have redundancy with the $\varepsilon$ for nuclear data uncertainties. The difficulty is to express these terms because they are evaluated by the experimenter in a first part and by the manufacturing uncertainty propagation in the second part. We consider that these two sources of uncertainties are not correlated and then, the Pearson coefficient between measurements and manufacturing data is null. Manufacturing data consist in all the data associated to the construction of the nuclear core such as mass balance, geometry of the core or position of elements of the core.

So, the expression of $\delta^{2}$, considering independent measurements from the manufacturing process of the core, is:

$$
\delta^{2}=\delta_{M D}^{2}+\delta_{M E A S}^{2}
$$

with:

$-\delta_{M D}^{2}$ represents the variances/covariances coming from Manufacturing Data (MD);

$-\delta_{M E A S}^{2}$ the uncertainty coming from the measurement method.

If we consider different experiments, these coefficients need to take into account correlations between measurements and manufacturing processes.
The evaluation of $\delta_{M D}^{2}$ can be performed using the method described in [15].

We assume we have experiments $E_{i}$ and $E_{j}$ and we want to write the correlations between manufacturing process of these two experiments. Then:

$$
\delta_{M D}^{2}\left(E_{i}, E_{j}\right)=\delta_{M D}\left(E_{i}\right) \delta_{M D}\left(E_{j}\right) r_{M D}\left(E_{i}, E_{j}\right) .
$$

For measurement uncertainties, the formulation is similar. The difficulty associated to these equations is the quantification of the Pearson correlations $r_{M D}\left(E_{i}, E_{j}\right)$ and $r_{M E A S}\left(E_{i}, E_{j}\right)$. A method has been proposed in [16].

To calculate the correlation factors, it is necessary in a first step to identify systematic uncertainties between $E_{i}$ and $E_{j}$ and statistical uncertainties. Systematic uncertainties are characterized by a correlation factor of 1 whereas statistical uncertainties have a correlation factor of 0 . Then, correlation factor can be estimated as follow:

$r_{M D}\left(E_{i}, E_{j}\right)=\frac{\left(\delta_{M D}\left(E_{i}\right) \delta_{M D}\left(E_{j}\right)\right)_{\text {systematic }}}{\sqrt{\left(\delta_{M D}\left(E_{i}\right) \delta_{M D}\left(E_{j}\right)\right)_{\text {systematic }}^{2}+\left(\delta_{M D}\left(E_{i}\right) \delta_{M D}\left(E_{j}\right)\right)_{\text {statistical }}^{2}}}$

The formulation is similar for measurement uncertainties.

Examples of systematic and statistical uncertainties are given in [16].

In the case of impossibilities to describe uncertainties as systematic and statistical, the following method is applied:

The value of $r_{M D}\left(E_{i}, E_{j}\right)$ can be estimated as follow:

- if the experiments $E_{i}$ and $E_{j}$ are performed on reactor cores which are manufactured in the same way, by the same manufacturer, we assume $r_{E_{i}, E_{j}}=1$;

- otherwise, if the reactor cores are built with different manufacturing processes, $\delta_{M D}^{2}\left(E_{i}, E_{j}\right)=0$.

To express measurement variances/covariances, we consider that the measures are fully correlated if the measurement technique for two experiments is based on the same process/measurement device. At the opposite, if the measurement techniques are different, we assume that the correlations are null. Then:

$\left\{\begin{array}{l}\delta_{M E A S}^{2}\left(E_{i}, E_{j}\right)=\delta_{M E A S}^{2}\left(E_{i}\right)=\delta_{M E A S}^{2}\left(E_{j}\right) \\ \text { if the } i \text { and } j \text { measurement techniques are the same } \\ \delta_{M E A S}^{2}\left(E_{i}, E_{j}\right)=0 \\ \text { if the } i \text { and } j \text { measurement techniques are different. }\end{array}\right.$

We can now express $\delta^{2}\left(E_{i}, E_{j}\right)$ with equations (7), (8) and (9) or with the strong assumption above.

In the next paragraph, we apply the adjustment system for multidimensional experiments.

\subsection{Expression of multidimensional system}

Now we consider we know $N$ integral parameters measurements $\left(E_{i}\right)_{1 \leq i \leq N}$ and the associated simulations $\left(C_{i}\right)_{1 \leq i \leq N}$ and we want to calculate the posterior 


$$
\begin{aligned}
& R^{*}-R:=\left[\begin{array}{c}
\vdots \\
R_{j}^{*}-R_{j} \\
\vdots
\end{array}\right] ;(E-C):=\left[\begin{array}{c}
\vdots \\
E_{i}-C_{i} \\
\vdots
\end{array}\right] \\
& M_{R}^{*}:=\left[\begin{array}{ccc}
\varepsilon^{2}\left(R_{1}\right) & \cdots & \varepsilon\left(R_{1}\right) \varepsilon\left(R_{Q}\right) r_{1, Q} \\
\vdots & \varepsilon^{2}\left(R_{j}\right) & \vdots \\
\varepsilon\left(R_{Q}\right) \varepsilon\left(R_{1}\right) r_{Q, 1} & \cdots & \varepsilon^{2}\left(R_{Q}\right)
\end{array}\right]^{*} \\
& S_{R} M_{\sigma} S_{E}{ }^{T}:=\left[\begin{array}{ccc}
S_{R_{1}} M_{\sigma} S_{E_{1}}{ }^{T} & \cdots & S_{R_{1}} M_{\sigma} S_{E_{N}}{ }^{T} \\
\vdots & S_{R_{j}} M_{\sigma} S_{E_{i}}{ }^{T} & \vdots \\
S_{R_{Q}} M_{\sigma} S_{E_{1}}{ }^{T} & \cdots & S_{R_{Q}} M_{\sigma} S_{E_{N}}{ }^{T}
\end{array}\right] \\
& {\left[M_{E}+S_{E} M_{\sigma} S_{E}^{T}\right]^{-1}:=\left[\begin{array}{ccc}
\delta_{1,1}^{2}+\varepsilon^{2}\left(E_{1}\right) & \cdots & \delta_{N, 1}^{2}+\varepsilon\left(E_{N}\right) \varepsilon\left(E_{1}\right) r_{N, 1} \\
\vdots & \delta_{i, i}^{2}+\varepsilon^{2}\left(E_{i}\right) & \vdots \\
\delta_{1, N}^{2}+\varepsilon\left(E_{1}\right) \varepsilon\left(E_{N}\right) r_{1, N} & \cdots & \delta_{N, N}^{2}+\varepsilon^{2}\left(E_{N}\right)
\end{array}\right]^{-1}}
\end{aligned}
$$

$$
\left\{\begin{array}{l}
R^{*}-R=\operatorname{diag}\left(\varepsilon\left(R_{j}\right)\right) C B^{-1} \operatorname{diag}\left(\varepsilon\left(E_{i}\right)\right)^{-1}\left[\begin{array}{c}
\vdots \\
E_{i}-C_{i} \\
\vdots
\end{array}\right] \\
M_{R}^{*}=M_{R}-\operatorname{diag}\left(\varepsilon\left(R_{j}\right)\right) C B^{-1} C^{T} \operatorname{diag}\left(\varepsilon\left(R_{j}\right)\right)
\end{array}\right.
$$

$$
\begin{aligned}
\text { where } B & =\left[\begin{array}{ccc}
\frac{\delta_{1,1}^{2}}{\varepsilon\left(E_{1}\right) \varepsilon\left(E_{1}\right)}+1 & \ldots & \frac{\delta_{N, 1}^{2}}{\varepsilon\left(E_{N}\right) \varepsilon\left(E_{1}\right)}+r_{E_{N}, E_{1}} \\
\vdots & \frac{\delta_{i, i}^{2}}{\varepsilon\left(E_{i}\right) \varepsilon\left(E_{i}\right)}+1 & \vdots \\
\frac{\delta_{1, N}^{2}}{\varepsilon\left(E_{1}\right) \varepsilon\left(E_{N}\right)}+r_{E_{1}, E_{N}} & \cdots & \frac{\delta_{N, N}^{2}}{\varepsilon\left(E_{N}\right) \varepsilon\left(E_{N}\right)}+1
\end{array}\right] \\
C & =\left[\begin{array}{ccc}
r_{E_{1}, R_{1}} & \cdots & r_{E_{N}, R_{1}} \\
\vdots & r_{E_{i}, R_{j}} & \vdots \\
r_{E_{1}, R_{Q}} & \cdots & r_{E_{N}, R_{Q}}
\end{array}\right]
\end{aligned}
$$

uncertainties of $Q$ integral parameters $\left(R_{j}\right)_{1} \leq j \leq Q$ (more general case). Starting with equation (4), we want to describe the system more precisely using matrix notation to make appear the Orlov coefficients $\omega$ and $r$ :

$$
\left\{\begin{array}{l}
R^{*}-R=S_{R} M_{\sigma} S_{E}^{T}\left[M_{E}+S_{E} M_{\sigma} S_{E}^{T}\right]^{-1}(E-C) \\
M_{R}^{*}=M_{R}-S_{R} M_{\sigma} S_{E}^{T}\left[M_{E}+S_{E} M_{\sigma} S_{E}^{T}\right]^{-1} S_{E} M_{\sigma} S_{R}^{T}
\end{array}\right.
$$

Detailing the matrix with indices, equation (4) can be written with the following notations:

$$
\text { See equations (10) above. }
$$

Multiplying equation (4) by

$$
\begin{aligned}
& \left(\operatorname{diag}\left(\varepsilon\left(R_{j}\right)\right) \operatorname{diag}\left(\varepsilon\left(R_{j}\right)\right)^{-1}\right)_{1 \leq j \leq Q} \\
& \operatorname{and}\left(\operatorname{diag}\left(\varepsilon\left(E_{i}\right)\right) \operatorname{diag}\left(\varepsilon\left(E_{i}\right)\right)^{-1}\right)_{1 \leq i \leq N}
\end{aligned}
$$

we transform the system as follow:

See equation (11) above.

Finally, in this equation we recognize the Orlov representativity $r_{E_{i}, R_{j}}$ in the matrix $C$ and the neutronic weight $\left(\frac{\delta_{\exp }^{2}}{\varepsilon\left(E_{i}\right) \varepsilon\left(E_{i}\right)}+1\right)^{-1}$ in the matrix $B$. If we come back to the assumptions of equation (5), considering one experiment and one application design, we find the monodimensional expression (Eq. (5)). 


$$
\begin{aligned}
& \left\{\begin{array}{l}
R^{*}-R=\operatorname{diag}\left(\sqrt{\varepsilon_{\sigma}^{2}\left(R_{j}\right)+\varepsilon_{F Y}^{2}\left(R_{j}\right)}\right) C B^{-1} \operatorname{diag}\left(\sqrt{\varepsilon_{\sigma}^{2}\left(E_{j}\right)+\varepsilon_{F Y}^{2}\left(E_{j}\right)}\right)^{-1}\left[\begin{array}{c}
\vdots \\
E_{i}-C_{i} \\
\vdots
\end{array}\right] \\
M_{R}^{*}=M_{R}-\operatorname{diag}\left(\sqrt{\varepsilon_{\sigma}^{2}\left(R_{j}\right)+\varepsilon_{F Y}^{2}\left(R_{j}\right)}\right) C B^{-1} C^{T} \operatorname{diag}\left(\sqrt{\varepsilon_{\sigma}^{2}\left(R_{j}\right)+\varepsilon_{F Y}^{2}\left(R_{j}\right)}\right)
\end{array}\right.
\end{aligned}
$$

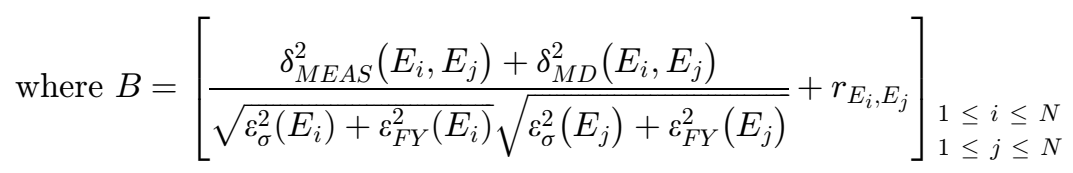

$$
\begin{aligned}
& C=\left(r_{E_{i}, R_{j}}\right) \begin{array}{l}
1 \leq i \leq N \\
1 \leq j \leq Q
\end{array} \\
& r_{X_{i}, Y_{j}}=\frac{\left[\begin{array}{l}
G_{X_{i}} \\
H_{X_{i}}
\end{array}\right]\left[\begin{array}{cl}
M_{\sigma} & 0 \\
0 & M_{F Y}
\end{array}\right]\left[\begin{array}{l}
G_{Y_{j}} \\
H_{Y_{j}}
\end{array}\right]^{T}}{\sqrt{\varepsilon_{\sigma}^{2}\left(X_{i}\right)+\varepsilon_{F Y}^{2}\left(X_{i}\right)} \sqrt{\varepsilon_{\sigma}^{2}\left(Y_{j}\right)+\varepsilon_{F Y}^{2}\left(Y_{j}\right)}}
\end{aligned}
$$

\section{Extension to coupled problem}

In the case where some experiments of the database are performed for example at different burnup to take into account the irradiation process, the method needs to be extended. Nuclide transmutation has to be added in the system. The new uncertainty terms coming from fission yield and transmutation have to be taken into account. These methods are described in [13] for transmutation terms, and in [14] for the fission yield uncertainties. Depletion perturbation theory [19] can also be used.

As shown in $[14,20]$, uncertainties of parameters of interests $\left(R_{j}\right)_{1 \leq j \leq Q}$ coming from cross-sections and fission yields for a coupled problem can be respectively written as:

$\left\{\begin{array}{l}M_{R, \sigma}=\left[S_{R}(D)+S_{R}(T) F_{\sigma}\right] M_{\sigma}\left[S_{R}(D)+S_{R}(T) F_{\sigma}\right]^{T}=G_{R} M_{\sigma} G_{R}{ }^{T} \\ M_{R, F Y}=\left[S_{R}(F Y) F_{F Y}\right] M_{F Y}\left[F_{F Y} S_{R}(F Y)\right]^{T}=H_{R} M_{F Y} H_{R}{ }^{T}\end{array}\right.$

with:

- $M_{R, \sigma}$ and $M_{R, F Y}$ the variances/covariances matrix of $\left(R_{j}\right)_{1 \leq j \leq Q}$ coming respectively from the cross-sections and from the fission yields;

- $S_{R}(D), S_{R}(T)$ and $S_{R}(F Y)$ the sensitivities matrix of $\left(R_{j}\right)_{1 \leq j \leq Q}$ to respectively the cross-sections (direct effect), the isotopic concentration (transmutation effect) and the fission yields as defined in [14];

- $F_{\sigma}$ and $F_{F Y}$ the Jacobian matrix describing the variations of isotopic concentrations respectively to variations of the cross-sections and the fission yields.

Restarting from equation (4),

$$
\left\{\begin{array}{l}
R^{*}-R=S_{R} M_{N D} S_{E}^{T}\left[M_{E}+S_{E} M_{N D} S_{E}^{T}\right]^{-1}(E-C) \\
M_{R}^{*}=M_{R}-S_{R} M_{N D} S_{E}^{T}\left[M_{E}+S_{E} M_{N D} S_{E}^{T}\right]^{-1} S_{E} M_{N D} S_{R}^{T}
\end{array}\right.
$$

with the new notation, $M_{N D}:=\left[\begin{array}{cc}M_{\sigma} & 0 \\ 0 & M_{F Y}\end{array}\right]$, $S_{R}:=\left[\begin{array}{c}G_{R} \\ H_{R}\end{array}\right]$ and $S_{E}$ is equivalent to $S_{R}$ but associated to the set of $E$ experiments and $M_{R}=M_{R, \sigma}+M_{R, F Y}$.

The total uncertainty $\varepsilon\left(E_{i}\right)$ of a neutronics parameter $E_{i}$ now depends on the cross-sections during core evolution and the fission yields. We consider they are independent because the correlations do not exist in the literature between cross sections and fission yields. The new (4) matricial system allows to take into account the isotopic composition of the core during time evolution.

In the case that some experiments represent an isotopic concentration evolution measurement, then the direct term $S_{R}(D)=S_{E}(D)=0$ and $S_{R}(T)=S_{R}(F Y)=I, I$ being the identity matrix. The matrix system (4) written adding this new information and performing the same transformations than from the previous paragraph becomes:

\section{See equation (13) above.}

The different quantities $S_{R}, S_{E}, \delta_{M D}^{2}, \varepsilon_{\sigma}^{2}, \varepsilon_{F Y}^{2}$ have to be calculated taking into account the evolution step of the core.

\section{Conclusions}

Accurate knowledge of nuclear data represents a major challenge in numerical simulations for reactor physics, since they are clearly identified as being the major source of propagated uncertainties on reactor integral parameters, such as reactivity, power distributions, and isotopic inventories. These nuclear data, and in particular microscopic cross sections, evaluated through differential experiments sometimes suffer from uncertainties currently not compatible with target uncertainties on some integral parameters, such as reactivity, reactivity effects, or reaction/transmutation rates. The combination of uncertainties propagation results described in [13-15], with inferential methods called "representativity" or 
"transposition", extended to the use of several integral parameters and with the results of experiments enables the re-estimation (sometimes still called adjustment) of cross sections of which remains to validate the effectiveness.

The construction of the posterior covariance matrix, i.e. taking into account measurement, is associated with adjusted cross sections that can be called posterior cross sections. On the other hand, the adjustment needed the consideration of the full set of cross sections which are impacting the interest parameters uncertainties [7]. Indeed, experimental data are collected relating to all cross sections since nuclear reactors are macroscopic systems configured by the substance they contain. These sections are all therefore affecting the quality of the result.

The extension of the inference method performed in this paper deals with two new items:

- the fuel depletion, which slightly modifies the variables used in the calculation of uncertainties;

- the cumulative uncertainty from transmutation effect. These uncertainties affect isotopic concentrations which in return modify the neutronics parameters of interest. If the parameter of interest is the isotopic concentration, then the direct effects are vanishing and some parameters of the equation do not have to be evaluated.

The second aspect to consider is the inclusion of measurements of local parameters as power factors or isotopic concentrations, which can reveal experimental correlations, because the measurement method is the same. It is then possible to extend the inference method to multiple and correlated experiments.

\section{Author contribution statement}

All the authors have contributed equally to the work presented in this manuscript.

\section{References}

1. V.V. Orlov, A.A. Van'kov, V.I. Voropaev, Y.A. Kazansky, V. I. Matveev, V.M. Murogov, Problems of fast reactor physics related to breeding, Energy Rev. 4, 991 (1980)

2. A. Gandini, On transposition of experimental reactor data to reference design, Technical report, Comitato nazionale per la ricerca e per lo sviluppo dell energia nucleare e delle energie alternative, 1983
3. P. Blaise, E. Fort, Resonance parameter adjustement methodoloby based on integral experiment analysis, Nucl. Sci. Eng. 133, 235 (1999)

4. T. Ivanova, E. Ivanov, F. Ecrabet, Uncertainty assessment for fast reactors based on nuclear data adjustment, Nucl. Data Sheets 118, 592 (2014)

5. C. De Saint Jean et al., Evaluation of cross section uncertainties using physical constraints: focus on integral experiments, Nucl. Data Sheets 123, 178 (2015)

6. E. Privas et al., Generation of $238 \mathrm{U}$ covariance matrices by using the integral data assimilation technique of the CONRAD code, EPJ Web Conf. 106, 04015 (2016)

7. G. Palmiotti et al., Combined use of integral experiments and covariance data, Nucl. Data Sheets 118, 596 (2014)

8. L.N. Usachev, Yu.G. Bokkov, INDC(CCP)-19/U, International Nuclear Data Committee, Vienna, 1972

9. G. Palmiotti, M. Salvatores, Use of integral experiments in the assessment of large liquid-metal fast breeder reactor basic design parameters, Nucl. Sci. Eng. 87, 333 (1984)

10. C. De Saint Jean, E. Dupont, M. Ishikawa, G. Palmioti, M. Salvatores, Assessment of existing nuclear data adjustment methodologies, Nuclear Science NEA/WPEC-33, 2010

11. P. Archier et al., COMAC, Nuclear data covariance matrices library for reactor applications, in Physor 2014

12. N. Terranova et al., Covariance generation and uncertainty propagation for thermal and fast neutron induced fission yields, EPJ Web Conf. 146, 02013 (2017)

13. T. Frosio, T. Bonaccorsi, P. Blaise, Nuclear data uncertainties propagation methods in Boltzmann/Bateman coupled problem: application to reactivity in MTR, Ann. Nucl. Energy 90, 303 (2016)

14. T. Frosio, T. Bonaccorsi, P. Blaise, Fission yields and cross section uncertainty propagation in Boltzmann/Bateman coupled problems: global and local parameters analysis with a focus on MTR, Ann. Nucl. Energy 98, 43 (2016)

15. T. Frosio, T. Bonaccorsi, P. Blaise, Manufacturing data uncertainties propagation method in burn-up problems, Sci. Technol. Nucl. Ins. 2017, 7275346 (2017)

16. Nuclear Science, Methods and issues for the combined use of integral experiments and covariance data, NEA/NSC/ WPEC/DOC(2013)445, 2013

17. M. Salvatores et al., Needs and issues of covariance data application, Nucl. Data Sheets 109, 2725 (2008)

18. N. Dossantos, Optimisation de l'approche de représentativité et de transposition pour la conception neutronique de programmes expérimentaux dans les maquettes critiques, PHD 2013, GRENI033

19. W.S. Yang, T.J. Downar, Depletion perturbation theory for the constrained equilibrium cycle, Nucl. Sci. Eng. 102, 365 (1989)

20. G. Palmiotti, M. Salvatores, Developments in sensitivity methodologies and the validation of reactor physics calculations, Sci. Technol. Nucl. Ins. 2012, 529623 (2012)

Cite this article as: Thomas Frosio, Thomas Bonaccorsi, Patrick Blaise, Extension of Bayesian inference for multi-experimental and coupled problem in neutronics - a revisit of the theoretical approach, EPJ Nuclear Sci. Technol. 4, 19 (2018) 